\title{
Alpha, Beta, Delta, Gamma: What's important to know about SARS-CoV-2 variants of concern?
}

\author{
Cite as: CMAJ 2021 July 12;193:E1059-60. doi: 10.1503/cmaj.1095949
}

Posted on cmajnews.com on June 18, 2020

$\mathrm{T}$ he World Health Organization recently announced a new naming system for SARS-CoV-2 variants using the Greek alphabet. The new naming system is aimed at preventing people from referring to the variants by the country where they were first detected. Four of these variants of concern - Alpha, Beta, Delta and Gamma - have spread to most provinces and territories in Canada.

\section{Alpha (B.1.1.7)}

The Alpha strain is the most common variant of concern in Canada, accounting for more than 216000 cases of COVID-19 across the country as of June 17.

First documented in the United Kingdom in September last year, Alpha is $50 \%$ more transmissible than earlier strains and was a major driver of Canada's third wave of COVID-19 in the first months of 2021.

The Alpha variant is also linked with surges in COVID-19 cases and hospitalizations among young people - although it's unclear whether those surges were due to the variant or other factors, says Dr. Gerald Evans, chair of infectious diseases at Queen's University in Kingston, Ont.

Daily reports from Public Health Ontario suggest new cases from the Alpha variant are declining. Evans attributes this drop to more than half of Canadians receiving at least one dose of a COVID19 vaccine.

One study found that the AstraZeneca vaccine was $70 \%$ effective in preventing symptomatic COVID-19 caused by the Alpha variant, while another study estimated the efficacy of the Pfizer vaccine at roughly $90 \%$.

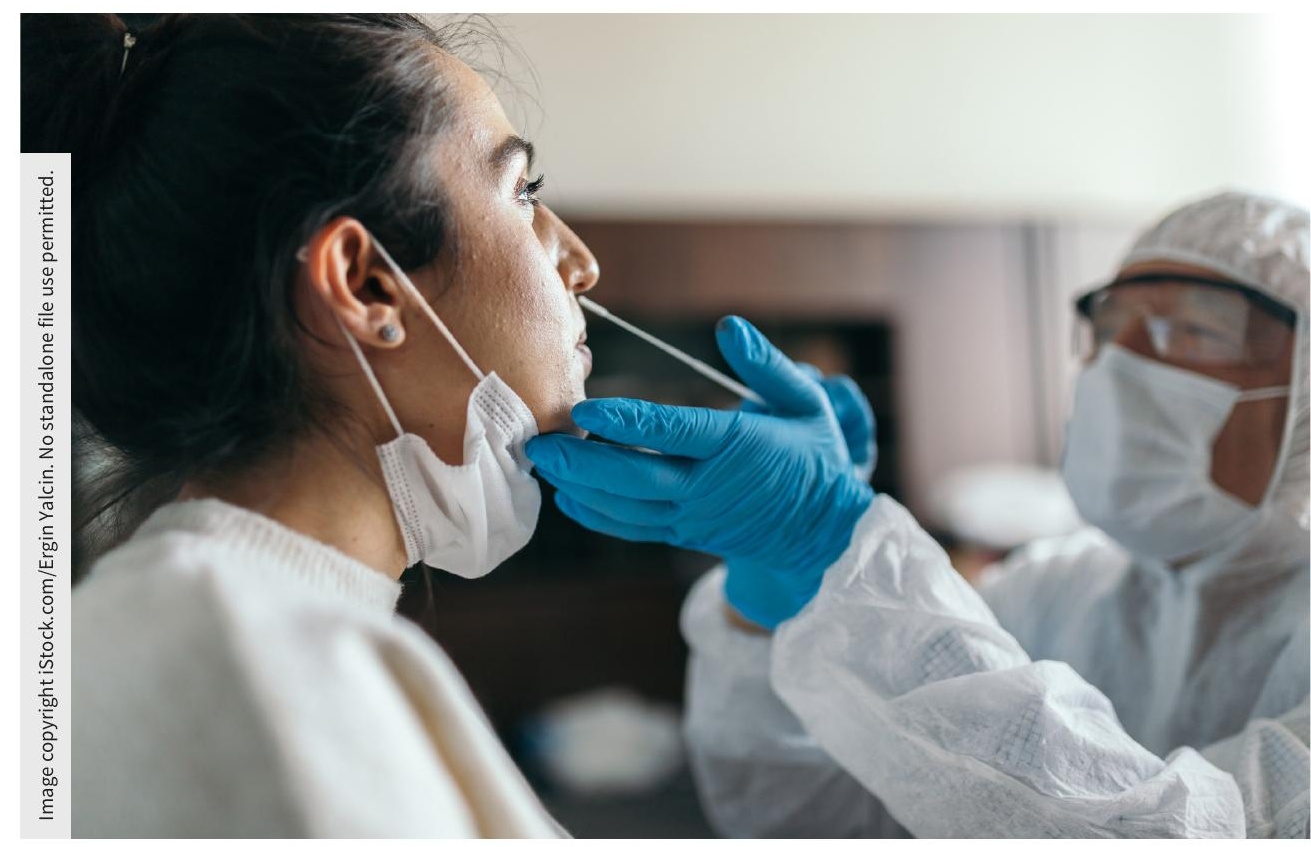

Canada's COVID-19 strategy may need to change in light of cases and deaths linked with the highly transmissible Delta variant.

\section{Delta (B.1.617.2)}

First documented in India in October last year, the highly transmissible Delta variant is on track to eclipse the Alpha variant as Canada's dominant strain this summer.

Studies out of the United Kingdom suggest the Delta variant is up to $60 \%$ more transmissible than the Alpha variant. Experts have warned the Delta strain may drive a fourth wave of COVID-19 in Canada and are urging governments to shift the focus of vaccination campaigns to deliver second doses as soon as possible.

According to Evans, Canada's case counts involving the Delta variant are unclear because the variant may not be detected by some rapid tests. Ontario estimates Delta accounted for $40 \%$ of new
COVID-19 cases in that province as of June 14. Alberta has reported several hundred cases and officials say the number of cases appears to be doubling every six to 12 days. Both Alberta and Manitoba have reported deaths linked to the Delta variant, including the death of at least one person who was fully vaccinated against COVID-19.

Vaccines appear to be less effective against the Delta variant than the Alpha strain, but they are still highly effective at preventing disease after two doses.

One study found that two doses of the Pfizer vaccine were $88 \%$ effective against symptomatic disease from the Delta variant, versus $93 \%$ effective against the Alpha strain. Two doses of the AstraZeneca were $60 \%$ effective against Delta compared to $66 \%$ against Alpha. 
However, both vaccines were only $33 \%$ effective against Delta after one dose, versus 50\% effective against Alpha.

Meanwhile, a preprint study reported high levels of protection when it came to hospitalizations for COVID-19. The Pfizer vaccine was 94\% effective against hospitalizations from the Delta or Alpha variants after one dose and $96 \%$ effective after two doses. The AstraZeneca vaccine was $71 \%$ effective against hospitalizations after one dose and 92\% effective after two.

According to Chief Public Health Officer, Dr. Theresa Tam, Canada may need to adjust COVID-19 vaccination targets to account for the Delta variant. Models that indicated that Canada could begin easing pandemic restrictions when $75 \%$ of people had at least one dose of a COVID19 vaccine did not account for the Delta strain.

Still, Canada is in a good position to curb the spread of the Delta variant, says Evans. "Because of our vaccine strategy to get one dose into everybody, the amount of SARS-CoV-2 circulating is really low," he explains. As such, the Delta variant "is not getting a good opportunity to surge forward."

\section{Beta (B.1.351)}

First documented in South Africa in May 2020, the Beta variant was linked with increases in hospitalizations and deaths during that country's second wave. Vaccines also appear to be less effective in preventing COVID-19 from the Beta variant.
In one study, researchers found that two doses of the Pfizer vaccine were $75 \%$ effective against any infection from the Beta variant, and $89.5 \%$ effective against any infection from the Alpha variant. However, vaccine effectiveness against severe or fatal disease from either the Alpha or Beta variants was very high at $97.4 \%$.

Novavax clinical trials showed $89 \%$ efficacy in the United Kingdom, compared to just $60 \%$ in South Africa, where the Beta strain was common. Similarly, trials of the Johnson \& Johnson vaccine reported lower levels of protection against moderate to severe COVID-19 in South Africa than in the United States.

Meanwhile, South Africa stopped plans to rollout the AstraZeneca vaccine because clinical trials did not show protection against mild or moderate illness caused by the Beta variant.

Recent studies suggest that mutations in the Beta variant's spike protein might allow it to escape the immune response trained by vaccines.

"Everybody was worried about Beta especially because there's this vaccine escape issue," says Evans. "If it was [more] transmissible like Alpha, we would have been in trouble, but it isn't. It seems to be no more transmissible [than early strains of the virus]." To date, Canada has documented more than 1900 cases.

\section{Gamma (P.1)}

The Gamma variant, first documented in Brazil in November 2020, is estimated to be 1.7-2.4 times more transmissible than other local strains in that country. Canada has reported more than 15000 cases to date.

The Gamma variant has some of the same mutations in its spike protein as the Alpha and Beta strains, which allow it to attach more easily to human cells. However, "it's not anywhere near as transmissible as Alpha or Delta," says Evans.

Previous infection with SARS-CoV-2 appears to provide less protection against reinfection with the Gamma variant than other strains. However, at least one preprint study showed that the Gamma variant is less resistant to antibody responses from previous illness or vaccination than the Beta variant.

Evidence also suggests that the Gamma variant struggles to compete with other strains in the wild. In a pre-print study, Italian researchers tracked the concurrent spread of Alpha and Gamma variants and found that the Alpha variant became increasingly common while the Gamma variant did not.

\section{Diana Duong, CMAJ}

Content licence: This is an Open Access article distributed in accordance with the terms of the Creative Commons Attribution (CC BY-NC-ND 4.0) licence, which permits use, distribution and reproduction in any medium, provided that the original publication is properly cited, the use is noncommercial (i.e., research or educational use), and no modifications or adaptations are made. See: https://creativecommons.org/ licenses/by-nc-nd/4.0/ 